\title{
XXI International Conference on Phosphorus Chemistry (ICPC 2016) Kazan, June 5-10, 2016
}

XXI International Conference on Phosphorus Chemistry (ICPC 2016) will be held on June, 5-10, 2016 in Kazan.

All fields of Phosphorus Chemistry will be considered: from fundamental to applied areas, including multidisciplinary approaches and related areas of other sciences.

\section{Organizers of the Conference}

Russian Academy of Sciences

Mendeleev Chemical Society of Republic of Tatarstan

Russian Scientific Foundation

Russian Foundation for Basic Research

Kazan (Volga Region) Federal University

Kazan National Research Technological

University
Arbuzov Institute of Organic and Physical Chemistry of RAS, Kazan

Kazan Scientific Center of RAS

Tatarstan Academy of Sciences

Tatarstan Regional Government

Ministry of Education and Science

of Russian Federation

Federal Agency for Scientific Organizations

\section{Conference Topics}

Progress in Organophophorus Chemistry;

New Phosphorus Reagents in Organic Synthesis;

Phosphorus in Coordination and Inorganic Chemistry;

Frontiers of Catalytic Processes;

Phosphorus in Life Sciences;

Towards New Age Materials Based on Phosphorus Compounds;

Phosphorus in Industry, Green Chemistry and Sustainable Development

School-conference "Rational Design of Phosphorus Substances with Desired Properties" will be held in the frame of the Conference.

Within each topic the targeted symposia, e.g. P-Stereogenic/P-Chirogenic symposium; Wittig symposium, Clusters and MOFs, Molecular Magnetizm, Luminescence, Antitumor Agents, etc., will take place on the Conference.

The Scientific Programm will include: plenary lectures (40 min); keynote lectures (30 $\mathrm{min})$; oral presentations (20 $\mathrm{min})$; flash presentations (5 $\mathrm{min})$; poster presentations.

Official Service Agent - International Agency of Congress Management.

Contact Information:

Tel.: +7 /495/ 13425 65. E-mail: info@icpc2016.ru; http://www.makongress.ru/eng/ 